\title{
L-DOPA PRELOADING INCREASES THE UPTAKE OF BOROPHENYLALANINE IN C6 GLIOMA RAT MODEL: A NEW STRATEGY TO IMPROVE BNCT EFFICACY
}

\author{
Silvia Capuani, Ph.D., ${ }^{* \dagger}$ Tommaso Gili, Ph.D., ${ }^{* \dagger}$ Marco Bozzali, M.D., ${ }^{\ddagger}$ Salvatore Russo, M.D. ${ }^{\S}$ \\ Paola Porcari, M.S., * Cesare Cametti, Ph.D., *₫ Emanuela D’Amore, M.S.," \\ Marco Colasanti, Ph.D., ** Giorgio Venturini, Ph.D., ${ }^{* *}$ Bruno Maraviglia, Ph.D. ${ }^{\dagger \uparrow}$ \\ Giuseppe Lazzarino, Ph.D. ${ }^{\dagger \dagger}$ and Francesco S. Pastore, M.D. ${ }^{\ddagger \ddagger}$
}

\begin{abstract}
*INFM-CNR CRS SOFT, Physics Department, “Sapienza” University of Rome, Italy; ${ }^{\dagger}$ Enrico Fermi Center, Rome, Italy; ${ }^{\ddagger}$ Neuroimaging Laboratory, Santa Lucia Foundation, Rome, Italy; ${ }^{\S}$ Department of Neurosurgery, Manchester University Hospital, Manchester, United Kingdom; “ Physics Department, "Sapienza” University of Rome, Italy; " Servizio Qualita'e Sieurezza della Sperimentazione Animale, Istituto Superiore di Sanità, Rome, Italy; ** Department of Biological Science, University "Rome III", Rome, Italy; ${ }^{\dagger \dagger}$ Department of Chemical Sciences, Laboratory of Biochemistry, University of Catania, Catania, Italy;
\end{abstract} and ${ }^{\ddagger \ddagger}$ Department of Neurosurgery, University “Tor Vergata”, Rome, Italy

\begin{abstract}
Purpose: Boron neutron capture therapy $(\mathrm{BNCT})$ is a radiotherapeutic modality based on ${ }^{10} \mathrm{~B}(\mathrm{n}, \alpha){ }^{7} \mathrm{Li}$ reaction, for the treatment of malignant gliomas. One of the main limitations for BNCT effectiveness is the insufficient intake of ${ }^{10} \mathrm{~B}$ nuclei in the tumor cells. This work was aimed at investigating the use of L-DOPA as a putative enhancer for ${ }^{10}$ B-drug 4-dihydroxy-borylphenylalanine (BPA) uptake in the C6-glioma model. The investigation was first performed in vitro and then extended to the animal model.

Methods and Materials: BPA accumulation in C6-glioma cells was assessed using radiowave dielectric spectroscopy, with and without L-DOPA preloading. Two L-DOPA incubation times ( 2 and 4 hours) were investigated, and the corresponding effects on BPA accumulation were quantified. C6-glioma cells were also implanted in the brain of 32 rats, and tumor growth was monitored by magnetic resonance imaging. Rats were assigned to two experimental branches: (1) BPA administration; (2) BPA administration after pretreatment with L-DOPA. All animals were sacrificed, and assessments of BPA concentrations in tumor tissue, normal brain, and blood samples were performed using high-performance liquid chromatography.

Results: L-DOPA preloading induced a massive increase of BPA concentration in C6-glioma cells only after

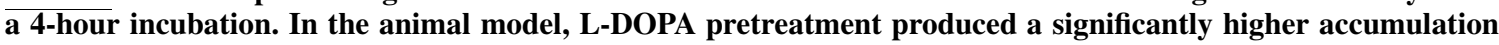
of BPA in tumor tissue but not in normal brain and blood samples.

Conclusions: This study suggests the potential use of L-DOPA as enhancer for BPA accumulation in malignant gliomas eligible for BNCT. L-DOPA preloading effect is discussed in terms of membrane transport mechanisms. (C) 2008 Elsevier Inc.
\end{abstract}

BNCT, C6-glioma, rat brain, L-DOPA, BPA, HPLC, MRI, Dielectric Spectroscopy.

\section{INTRODUCTION}

Malignant gliomas are the most common primary intracranial neoplasms in humans, accounting for about $78 \%$ of all malignances of the central nervous system (1). More than $80 \%$ of these tumors are considered high-grade (Grades 3 and 4) according to the current World Health Organization criteria (2). Anaplastic astrocitomas (Grade 3) and glioblastomas (Grade 4) are typically associated with a severe prognosis, with a median overall survival of 1-5 years (2). Despite advances in microsurgical techniques, radiotherapy, and chemotherapy, there has been little improvement in the clinical outcome of patients suffering from these kinds of tumors.
Boron Neutron Capture Therapy (BNCT) (3-6) is a technique based on a targeted radiation approach, which represents an alternative adjuvant therapy for malignant gliomas. It has already been used in patients with various types of brain malignances, including glioblastomas (7-12), anaplastic meningiomas (13), cerebral melanoma metastases (14), or tumors recurrences in inoperable anatomic locations $(15,16)$. In this context, a few Phase I $(7,8,10,11)$ and Phase II studies $(8,10)$ have consistently demonstrated no severe effects of BNCT-related toxicity, and some preliminary evidence of therapeutic effectiveness $(7,9,12)$ has been presented. From a technical point of view, BNCT is based on the
Reprint requests to: Dr. Silvia Capuani, Physics Department University "La Sapienza," Piazzale Aldo Moro 2, 00185 Rome, Italy. Tel/Fax: (+39) 06-49913928; E-mail: silvia.capuani@roma1.infn.it
Conflict of interest: none.

Received March 31, 2008, and in revised form June 11, 2008. Accepted for publication June 11, 2008. 
intratumoral delivery of a stable boron isotope $\left({ }^{10} \mathrm{~B}\right.$ nucleus $)$ featuring high cross-section for thermal neutrons capture. In the presence of an adequate amount of ${ }^{10} \mathrm{~B}$ within the tumor cells (approximate minimal effective dose: $20-35 \mu \mathrm{g} / \mathrm{g}$ ), irradiation with low-energy (thermal or epithermal) neutron particles $\left({ }^{10} \mathrm{~B}+\mathrm{n}_{\mathrm{th}} \rightarrow \alpha+{ }^{7} \mathrm{Li}+2.79 \mathrm{MeV}\right)$ induces a short-range nuclear reaction. As a consequence, heavy charged particles $\left({ }^{7} \mathrm{Li}\right.$ and $\alpha$ particles) characterized by high linear energy transfer (LET) produce a disruptive effect within a spatial range of $5-10 \mu \mathrm{m}$. These characteristics ensure a well-localized effect of BNCT, which is virtually confined to the cells containing a critical amount of ${ }^{10} \mathrm{~B}$. A more selective and higher accumulation of ${ }^{10} \mathrm{~B}$ in the tumor cells will result in a more effective and less toxic BNCT. As mentioned earlier, some clinical trials have reported encouraging results on the use of BNCT in patients with glioblastomas. However, the clinical outcome is still considered largely unsatisfactory. It is general opinion that one of the major limitations for BNCT effectiveness is the insufficient incorporation of ${ }^{10} \mathrm{~B}$ into the tumor cells, even considering the most advanced methods of ${ }^{10} \mathrm{~B}$ administration (17-20). An additional limitation of BNCT is the relatively low specificity of ${ }^{10} \mathrm{~B}$ uptake in tumor cells as compared with the surrounding normal tissues.

Thus far, two ${ }^{10} \mathrm{~B}$ carriers have been used for clinical purposes: the mercapto-undecahydrododecaborate, $\mathrm{Na}_{2} \mathrm{~B}_{12} \mathrm{H}_{11} \mathrm{SH}$ (BSH) (21) and the p-dihydroxyboryl-phenylalanine $\mathrm{C}_{9} \mathrm{H}_{12} \mathrm{BNO}_{4}$ (BPA) (22). A large body of literature has demonstrated a higher effectiveness of BPA compared with BSH in the BNCT of brain malignances $(17,23,24)$. This might be explained by a different microdistribution and tumor incorporation of the two compounds (25-28). BPA is believed to pass through the blood brain barrier (BBB) and cell membranes, and it is found at higher concentrations in tumor cells (29). The details of the uptake mechanisms for BPA into tumor cells are still not completely understood. There is evidence that such an uptake is supported by a carrier-mediated transport rather than by passive diffusion. Some authors have demonstrated that the administration of L-tyrosine increases the intracellular accumulation of BPA in mouse melanoma cells (30). This effect has also been replicated in 9L rat gliosarcoma cells (31). Moreover, BPA accumulation in 9L rat gliosarcoma cells was enhanced by either pretreatment with molecules targeted by L or A aminoacid transport systems. These findings suggest that such transporters work with a substratecoupled antiport (exchange) mechanism, which is enhanced by preloading of specific aminoacids. L-DOPA is a wellknown molecule with a chemical structure similar to those of L-tyrosine and BPA. A previous in vitro study on 9L rat gliosarcoma cells has demonstrated that L-DOPA preloading improves several times the intracellular accumulation of BPA (31). Conversely, a simultaneous incubation of 9L gliosarcoma cells with L-DOPA and BPA causes a decrease of intracellular BPA accumulation. These observations are particularly interesting not only for biological speculation but also for their impact on potential clinical applications.
In fact, the large experience accumulated in the use of L-DOPA (at different doses) as a treatment for Parkinson disease $(32,33)$ might allow its employment as a potential enhancer of BPA accumulation in BNCT clinical trials for malignant gliomas.

These considerations prompted the current work, which focused on investigating the potential role of L-DOPA in BNCT. We adopted for our experiments the C6 glioma cell line, which has been widely used to evaluate in vitro the effects of novel therapies and to produce animal models based on tumor-cell implantation. Specific aims of this study were as follows: (1) to replicate in C6 glioma cells the findings previously described in 9L gliosarcoma cells (31) by demonstrating a significant increase of BPA intracellular accumulation due to L-DOPA preloading (Experiment 1); (2) to assess in vivo, using the C6 glioma cell rat model, the effect of L-DOPA preloading on BPA accumulation in tumors as compared to normal brain tissue (Experiment 2). Experiment 1 was conducted using radiowave dielectric spectroscopy, which allows passive electric parameters of cell membrane to be measured (34) - namely, the permittivity $\left(\varepsilon_{\mathrm{s}}\right)$ and the electrical conductivity $\left(\sigma_{\mathrm{s}}\right)$ of the cell membrane and the electrical conductivity $\left(\sigma_{\mathrm{p}}\right)$ of the intracellular medium (cytosol). Changes of $\sigma_{\mathrm{p}}$ have been shown as proportional to variations in intracellular BPA content (27). In Experiment 2, BPA quantification in animal tissues was performed using high performance liquid chromatography (HPLC). As previously shown, this technique is a reliable method for analysis of BPA incorporation in several biological tissues, including cerebral samples (35).

\section{METHODS AND MATERIALS}

\section{Experiment 1}

This experiment was performed by radiowave dielectric spectroscopy, adopting the same procedure previously employed to assess the intracellular accumulation of BPA and BSH in C6-glioma cells (27). A detailed description about cell cultures and radiowave spectroscopy measurements may be found elsewhere $(27,34)$. Briefly, C6 glioma cells were grown in Dulbecco's modified Eagle's medium supplemented with $10 \%$ fetal bovine serum and $50 \mu \mathrm{g} /$ $\mathrm{mL}$ of gentamicin at $37^{\circ} \mathrm{C}$ under an atmosphere of $5 \% \mathrm{CO}_{2}$ in air. Each sample included about $1.6 \times 10^{8}$ cells. The first step was to generate a reference sample to assess the basic characteristics of permittivity and electrical conductivity $\sigma_{\mathrm{s}}$ of the C6 glioma cell membranes. Equivalent samples were then produced to investigate changes of the electrical conductivity $\sigma_{\mathrm{p}}$ under five experimental conditions: (l) cells incubated with addition of $2 \mathrm{mmol}$ BPA (Condition 1); (2) cells incubated with addition of $50 \mu \mathrm{g} / \mathrm{mL}$ of L-DOPA for 2 hours (Condition 2); (3) cells incubated with addition of $50 \mu \mathrm{g} /$ $\mathrm{mL}$ of L-DOPA for 4 hours (Condition 3); (4) cells incubated with addition of $2 \mathrm{mmol}$ BPA after a 2-hour preincubation with $50 \mu \mathrm{g} / \mathrm{mL}$ L-DOPA (Condition 4); (5) cells incubated with addition of $2 \mathrm{mmol}$ BPA after a 4-hour preincubation with $50 \mu \mathrm{g} / \mathrm{mL}$ L-DOPA (Condition 5). Before any dielectric measurement, each sample was washed twice with fresh serum-free culture medium. Dielectric measurements were carried out at $37^{\circ} \mathrm{C}$, by evaluating permittivity $\varepsilon(\omega)$ and conductivity $\sigma(\omega)$ of whole cell suspensions in a frequency range from $1 \mathrm{kHz}$ to $1 \mathrm{GHz}$. The conductivity value of the cytosol $\left(\sigma_{\mathrm{p}}\right)$ was obtained by fitting, within the effective medium theory 
approximation (36), an appropriate mixture equation based on a single-shell model to the experimental $\varepsilon(\omega)$ and $\sigma(\omega)$ spectra. This parameter, which probes the whole ionic content within the cytosol, is influenced by the intracellular concentration of BPA, L-DOPA, or both. For each experiment or condition, conductivity cytosol changes were reported as percentage differences $\left(\sigma_{\mathrm{p}}{ }^{\text {test }}-\sigma_{\mathrm{p}}{ }^{\text {ref }}\right) / \sigma_{\mathrm{p}}{ }^{\text {ref }}$ between values measured in the test- $\left(\sigma_{\mathrm{p}}^{\text {test }}\right)$ and in the referencesample $\left(\sigma_{\mathrm{p}}{ }^{\text {ref }}\right)$, respectively.

\section{Experiment 2}

Thirty-two male Wistar rats weighing 300-350 g were used for the current experiment. All procedures related to animal care were performed in accordance with Decree 116/92, which represents the Italian enforcement of the European Directive 86/609/EEC. In the first step of Experiment 2, each rat, intraperitoneally anesthetized with chloralium hydrate $(1.5 \mathrm{mg} / \mathrm{Kg})$, underwent brain implantation of partially deantigenized C6 glioma cells. The head of each rat was held into a stereotaxic frame (produced by Stoelting, IL), and a burr hole $(3 \mathrm{~mm}$ ) was made using a hand drill at the right calvarium (stereotaxic coordinates: $2 \mathrm{~mm}$ anterior to the right coronal suture and $5 \mathrm{~mm}$ to the right of the midline). Culture medium (10 $\mu \mathrm{L})$ containing $10^{6}$ cells was then inoculated at a depth of $4 \mathrm{~mm}$ from the dural surface using a Hamilton syringe with a 26-gauge needle. The needle was slowly removed 2 min after the injection. The burr hole was covered with candle wax, and the scalp was sutured. No complications were encountered during the surgical procedure. Brain MRI monitoring was used to assess tumor implantation and growth in each rat. Five days after surgical implantation of C6 glioma cells, each rat underwent a first MRI scan (baseline) using a system operating at 7T (Bruker Biospec 70/15, Eitlingen, Germany), equipped with a 40 -mm-diameter ${ }^{1} \mathrm{H}$ surface coil. Rats were reanesthetized with chloralium hydrate $(1.5 \mathrm{mg} / \mathrm{Kg})$ and positioned on a homemade bed with the head secured to the surface coil. T1-weighted (repetition time/echo time $=1200 / 12.9 \mathrm{~ms}$ ) and T2-weighted (repetition time/echo time $=2500 / 40 \mathrm{~ms}$ ) Spin Echo sequences were acquired with a $128 \times 128$ matrix size, a $40-\mathrm{mm}$ field of view, and a $312 \times$ $312 \mu \mathrm{m}$ of in plane image resolution. In both sequences, four contiguous 1.5-mm-thick slices were collected to cover the entire brain. Using the same acquisition protocol, all rats underwent serial MRI assessments (once every 4 days) for longitudinal monitoring of tumor growth. When the tumor size reached a minimum diameter of $2.0 \mathrm{~mm}$ on T2-weighted images, rats were randomly assigned to one of the two experimental branches: (1) BPA administration with L-DOPA pretreatment or (2) BPA administration without LDOPA pretreatment (control group). Animals belonging to the first branch $(n=16)$ received $50 \mathrm{mg} / \mathrm{Kg}$ L-DOPA intraperitoneally 24 hours before BPA administration. This large dose of L-DOPA and the long time gap were chosen to obtain a prolonged LDOPA availability than that observed at lower doses (37), with negligible competition effects for BBB transport between L-DOPA and its metabolites and for BPA. Indeed, a 15-hour half-life has been estimated for the 3-MT metabolite in rats treated with L-DOPA (38). Each rat was then anesthetized again as described earlier, and its right internal carotid was catheterized using a $0.7-\mathrm{mm}$ cannula. This access was immediately used for the infusion of $300 \mathrm{mg} / \mathrm{Kg}$ BPA-fructose complex, administered over $30 \mathrm{~min}$ by a constant flow pump (Harvard Apparatus, Cambridge, MA). Animals belonging to the control branch $(n=16)$, underwent the same procedure (right internal carotid incannulation, infusion of BPA-fructose complex) with no preadministration of L-DOPA. Seven animals died from complications during the carotid infusion of BPA. The 25 survivors (15 from branch 1 and 10 from branch 2) were sacrificed by craniotomy 150 min after BPA infusion because at this time the higher tumor-to-ipsilateral brain BPA ratios are expected (39). Tumor tissue, normal brain (obtained from both hemispheres, ipsi- and contra-lateral to the tumor implantation), and blood samples were collected for HPLC analysis. HPLC procedure for BPA quantification was performed as previously described (35). Briefly, the reversed-phase HPLC method exploits BPA derivatization with OPA and a spectrofluorimetric detector. The lowest BPA detection limit of this approach is 0.5 pmol.

\section{Statistical analysis}

Experiment 1. Statistical evaluation of the effect of L-DOPA preloading was made by analysis of variance (ANOVA) to test for differences among groups (Conditions 1 to 5). Then, a post hoc analysis, using the Student-Newman-Keuls method [SKN test], was applied for between group comparisons at a significant level of $p<0.05$ when a significant difference was detected by ANOVA.

Experiment 2. The means and SD were computed for BPA concentrations measured from the following samples obtained from each studied animal: (1) tumor tissue; (2) normal brain tissue, collected in both cerebral hemispheres (ipsi- and contralateral to C6 glioma cells implantation); (3) blood. A Student $t$ test for nonpaired data was employed to investigate group differences between those animals which received L-DOPA pretreatment and those animals that did not. To correct for multiple comparisons and to minimize the risk of type II errors, only $p$ values $\leq 0.001$ were considered statistically significant).

\section{RESULTS}

\section{Experiment 1}

In Fig. 1 , mean ( \pm SD) percentage differences between $\sigma_{\mathrm{p}}$ values measured in test and in reference samples are reported for each condition. The ANOVA analysis including the five experimental conditions (described earlier) revealed the presence of significant differences among them. SKN test demonstrated significantly higher $\sigma_{\mathrm{p}}$ percentage difference values in Condition 5 (BPA addition after 4-hour L-DOPA incubation) compared with any other condition. Moreover, significantly higher $\sigma_{\mathrm{p}}$ percentage difference values were found in Condition 3 (4-hour L-DOPA incubation) compared with Condition 2 (2-hour L-DOPA incubation). To exclude that the $\sigma_{\mathrm{p}}$ percentage difference observed in Condition 5 was the result of an independent accumulation of L-DOPA and BPA at two different times, we performed the following additional comparison: [mean $\sigma_{\mathrm{p}}$ percentage difference observed in Condition 5] vs [ $\sigma_{\mathrm{p}}$ percentage difference observed in Condition 1$)+\left(\sigma_{\mathrm{p}}\right.$ percentage difference observed in condition 3)]. The statistical result showed significantly higher $\sigma_{\mathrm{p}}$ percentage values in Condition 5 than in Condition 1 and Condition 3.

\section{Experiment 2}

All rats survived the surgical implantation of C6 glioma cells. As shown by MRI data collected 5 days after operation (baseline), all animals developed a brain tumor with an average diameter of $1 \mathrm{~mm}$. Longitudinal MRI monitoring demonstrated tumor growth in all rats. On average, tumor lesions reached a diameter $\geq 2 \mathrm{~mm} 12$ days after surgical 


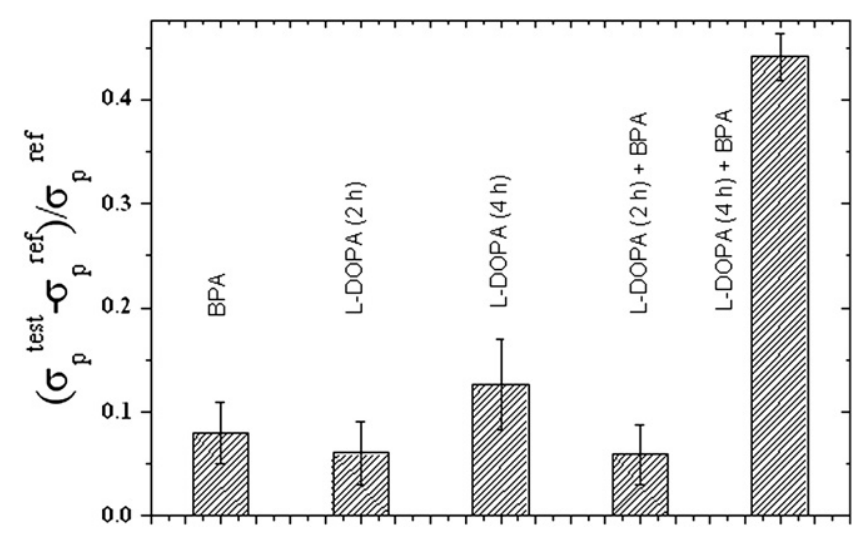

Fig. 1. Effects of L-DOPA preloading on borylphenylalanine (BPA) intracellular accumulation in C6 glioma cells. Percentage changes in cytosol electrical conductivity $\left(\sigma_{\mathrm{p}}\right)$ obtained from C6 glioma cells under five experimental conditions: $(1)$ cells incubated with addition of 2 mmol BPA (first column on the left); (2) cells incubated with addition of $50 \mu \mathrm{g} / \mathrm{mL}$ of L-DOPA for 2 hours (second column from the left); (3) cells incubated with addition of $50 \mu \mathrm{g} / \mathrm{mL}$ of L-DOPA for 4 hours (third column from the left); (4) cells incubated with addition of 2 mmol BPA after a 2-hour preincubation with $50 \mu \mathrm{g} / \mathrm{mL}$ L-DOPA (forth column from the left); (5) cells incubated with addition of $2 \mathrm{mmol}$ BPA after a 4-hour preincubation with $50 \mu \mathrm{g} / \mathrm{mL}$ L-DOPA BPA (fifth column from the left). Values are expressed as mean $( \pm \mathrm{SD})$ percentage difference of $\sigma_{\mathrm{p}}$ between each test sample (C6 glioma cells under a specific experimental condition) and the reference samples. Significantly higher $\sigma_{\mathrm{p}}$ percentage difference values were observed in Condition 5 (BPA addition after 4-hour L-DOPA incubation) compared with any other experimental condition, and in Condition 3 (4-hour L-DOPA incubation) compared with Condition 2 (2-hour L-DOPA incubation). Group comparisons did not show any additional significant difference. See text for further details.

implantation (range, 10-14 days). The mean (SD) tumor volume assessed on $\mathrm{T} 2-\mathrm{w}$ scans from all the studied rats was $55.8(8.3) * 10^{-3} \mathrm{~mL}$.

BPA concentrations assessed by HPLC from each sampled tissue (tumor, normal brain, blood) are summarized in Table 1 for the two experimental groups: rats that received BPA infusion only (Group A) and rats that received L-DOPA pretreatment before BPA infusion (Group B). BPA accumulation in tumor samples was significantly higher in Group B compared with Group A $(p<0.0001)$. Conversely, no significant difference was found in normal brain (sampled in both cerebral hemispheres) and blood samples between the two animal groups.

\section{DISCUSSION}

In this work, we tested the hypothesis that L-DOPA has an active and selective role in increasing the intracellular accumulation of BPA in C6 glioma cells. The results obtained in vitro demonstrate that L-DOPA promotes the cellular uptake of BPA, and extend findings previously observed in 9L gliosarcoma cells (31). As suggested by other authors (31), such an effect is likely to be related to mechanisms of active membrane transport, which are triggered by specific conditions. Indeed, our experiments showed that the $\sigma_{\mathrm{p}}$
Table 1. BPA accumulation in sample of tumor tissue, normal brain, and blood from C6-glioma animal models with and without L-DOPA pretreatment

\begin{tabular}{lcc}
\hline & \multicolumn{2}{c}{$\begin{array}{c}\text { Mean } \pm \text { SD BPA/tissue } \\
\text { concentration }(\mu \mathrm{g} / \mathrm{g})\end{array}$} \\
\hline Sample & $\begin{array}{c}\text { Group A }(n=10) \\
\text { Tumor }\end{array}$ & $\begin{array}{c}\text { Group B }(n=15) \\
\text { Normal brain } \\
\quad \text { (ipsilateral) }\end{array}$ \\
$\begin{array}{c}\text { Normal brain } \\
\text { (contralateral) }\end{array}$ & $12.0 \pm 5.5$ & $10.6 \pm 6.2$ \\
Blood & $7.4 \pm 2.2$ & $4.8 \pm 2.1$ \\
\hline
\end{tabular}

Abbreviations: $\mathrm{BPA}=$ borylphenylalanine; Group $\mathrm{A}=$ animals that received $\mathrm{BPA}$ infusion only; Group $\mathrm{B}=$ animals that received L-DOPA administration 24 hours before BPA infusion.

BPA concentration assessed by high-performance liquid chromatography from the sampled tissues (tumor, normal brain, blood) obtained from the C6 cells glioma rat model under two experimental conditions: BPA infusion (Group A); BPA infusion with L-DOPA pretreatment (Group B). Normal brain tissue was sampled from both hemispheres, ipsilateral and contralateral to glioma cells implantation. BPA concentration in tumor samples was significantly higher in condition B than in condition A. Conversely, no significant difference was found in normal brain and blood samples between the two groups.

changes resulting from the single addition of L-DOPA or BPA to cell cultures, is lower than that observed when the two compounds are given in combination (Fig. 1). Moreover, the latter effect is dependent on timing modalities by which the two compounds are administered. L-DOPA preloading increased 5 times the BPA intracellular accumulation with 4-hour incubation, whereas no significant change was observed with 2-hour preloading (Fig. 1). These findings indicate that the enhancing effect of L-DOPA preloading is based on events occurring in a relatively long time. Previous work has proposed that both BPA and L-DOPA penetrate through the cell membrane using two main mechanisms: by diffusion (slow process, essentially driven by concentration gradients) and by active carries (fast process). Previous experiments, using mouse melanoma cells, suggested the presence of specific membrane antiport carriers with an high affinity for L-substrates, such as L-tyrosine, L-BPA, and L-DOPA (31). The activation of these carriers is supposed to be driven by a chemical gradient of L-molecules across cell membranes, due to their preliminary accumulation inside the cytosol (31). Using our results, we can speculate that 4 hours are necessary to reach in C6-glioma cells, by slow diffusion, a critical intracellular concentration of LDOPA to trigger the faster L-antiport system. This explains why a massive accumulation of BPA was observed in C6 glioma cells only after 4-hour L-DOPA preloading.

The most striking findings of this work are the convergent results obtained with C6 glioma cells in vitro and using the equivalent animal model. L-DOPA preadministration produced in the rat model an enhancement of tumor BPA accumulation, which was about 2.7 times higher than in the control condition (Table 1). In clinical application, one of 
the main limitations for BNCT effectiveness is the insufficient accumulation of ${ }^{10} \mathrm{~B}$ carrier into the tumor cells. In this perspective, our results are particularly encouraging and might be considered for future BNCT clinical trials in humans. When comparing BPA concentration in blood and normal brain, there was no significant difference between rats that received L-DOPA and rats that did not. This makes the potential use of L-DOPA in BNCT of brain tumors even more attractive. Indeed, these results show the potential ability of L-DOPA to induce a significant increase of BNCT effectiveness (i.e., tumor cells disruption) without remarkable side effects (i.e., normal brain tissue damage) associated. The selective effect of L-DOPA on tumor tissues is likely to be due to genetic abnormalities in C6-glioma cells, conditioning an overexpression of antiport membrane carriers for $\mathrm{L}$ substrates.

Thus far, other strategies have been proposed to improve BNCT effectiveness by increasing BPA or BSH tumor accumulation. Some authors employed agents such as Cereport (RMP-7) (40) or mannitol $(40,41)$ to induce BBB disruption in different tumor models. Other researches focused on different modalities of BPA administration, demonstrating higher BPA concentrations in the L9-glioblastoma model after a prolonged intracarotid BPA infusion (6 hours vs 2 hours) $(42,43)$. These alternative strategies showed an increase of indexes such as tumor:brain and tumor:blood BPA concentration, suggesting a potential usefulness for BNCT. However, their potential toxicity has not been fully investigated. Our study showed a remarkable and selective increase of BPA uptake in tumor tissues using L-DOPA, which is a well-tolerated medication associated with modest side effects and low toxicity.

To the best of our knowledge, there are no published studies that have tested in vivo the effect of L-DOPA on BPA uptake in brain tumors. Our results appear promising, especially for their potential application to clinical therapeutic protocols. Nevertheless, this study has several limitations, such as the lack of determination of L-DOPA and BPA concentrations from blood, tumor, and normal brain samples at different time points and the absence of assessment of the radiation effects. Further animal studies are therefore needed not only to investigate further the effect of L-DOPA pretreatment on tumor BPA accumulation but also to evaluate the final result that may be obtained after BNCT radiation. In particular, appropriate protocols should be defined in terms of modalities of L-DOPA and BPA administration (i.e., doses and timing). These insights might eventually promote pilot studies in patients with malignant gliomas who are eligible for BNCT.

\section{REFERENCES}

1. Central Brain Tumor Registry of the United States. Statistical Report: Primary Brain Tumors in the United States, 1998-2002. Hinsdale, IL: Central Brain Tumor Registry of the United States; 2006.

2. Kleihues P, Cavenee WK. Pathology and Genetics of Tumors of the Nervous System (World Health Organisation Classification of Tumours). Lyon, France: IARC Press; 2000. p.314.

3. Sauerwein W. Principles and history of neutron capture therapy. Strahlenther Onkol 1993;169:1-6.

4. Barth RF, Soloway AH, Goodman JH, et al. Boron neutron capture therapy of brain tumors: An emerging therapeutic modality. Neurosurgery 1999;44:433-450.

5. Barth RF, Coderre JA, Vicente MG, Blue TE. Boron neutron capture therapy of cancer: current status and future prospects. Clin Cancer Res 2005;11:3987-4002.

6. Yamamoto T, Nakai K, Matsumura A. Boron neutron capture therapy for glioblastoma. Cancer Lett 2008;262:143-152.

7. Capala J, Stenstam BH, Sköld K, et al. Boron neutron capture therapy for glioblastoma multiforme: Clinical studies in Sweden. J Neurooncol 2003;62:135-144.

8. Busse PM, Harling OK, Palmer MR, et al. A critical examination of the results from the Harvard-MIT NCT program Phase I clinical trial of neutron capture therapy for intracranial disease. J Neurooncol 2003;62:111-121.

9. Joensuu H, Kankaanranta L, Seppälä T, et al. Boron neutron capture therapy of brain tumors: Clinical trials at the finnish facility using boronophenylalanine. J Neurooncol 2003;62: 123-134.

10. Diaz AZ. Assessment of the results from the Phase I/II boron neutron capture therapy trials at the Brookhaven National Laboratory from a clinician's point of view. J Neurooncol 2003;62: 101-109.

11. Palmer MR, Goorley JT, Kiger WS, et al. Treatment planning and dosimetry for the Harvard-MIT Phase I clinical trial of cranial neutron capture therapy. Int J Radiat Oncol Biol Phys 2002; 53:1361-1379.
12. Stenstam BH, Pellettieri L, Sorteberg W, et al. BNCT for recurrent intracranial meningeal tumours-case reports. Acta Neurol Scand 2007; 115:243-247.

13. Miyatake S, Tamura Y, Kawabata S, et al. Boron neutron capture therapy for malignant tumors related to meningiomas. Neurosurgery 2007;61:82-90.

14. Coderre JA, Turcotte JC, Riley KJ, et al. Boron neutron capture therapy: Cellular targeting of high linear energy transfer radiation. Technol Cancer Res Treat 2003;2:355-375.

15. Kato I, Ono K, Sakurai Y, et al. Effectiveness of BNCT for recurrent head and neck malignancies. Appl Radiat Isot 2004; 61:1069-1073.

16. Kankaanranta L, Seppälä T, Koivunoro H, et al. Boron neutron capture therapy in the treatment of locally recurred head and neck cancer. Int J Radiat Oncol Biol Phys 2007;69:475-482.

17. Coderre JA, Glass JD, Fairchild RG, et al. Selective targeting of boronophenylalanine to melanoma in BALB/c mice for neutron capture therapy. Cancer Res 1987;47:6377-6383.

18. Elowitz EH, Bergland RM, Coderre JA, et al. Biodistribution of p-boronophenylalanine in patients with glioblastoma multiforme for use in boron neutron capture therapy. Neurosurgery 1998;42:463-468.

19. Chadha M, Capala J, Coderre JA. Boron neutron-capture therapy (BNCT) for glioblastoma multiforme (GBM) using the epithermal neutron beam at the Brookhaven National Laboratory. Int J Radiat Oncol Biol Phys 1998;40:829-834.

20. Chanana AD, Capala J, Chadha M, et al. Boron neutron capture therapy for glioblastoma multiforme: Interim results from the Phase I/II dose-escalation studies. Neurosurgery 1999;44: 1182-1192.

21. Soloway AH, Hatanaka H, Davis MA. Penetration of brain and brain tumor. VII. Tumor-binding sulfhydryl boron compounds. J Med Chem 1967;10:714-717.

22. Snyder HR, Reedy AJ, Lennarz WJ. Synthesis of aromatic boronic acids, aldehyde boronic acids and a boronic acid analog of tyrosine. J Am Chem Soc 1958;80:835-838. 
23. Ono K, Masunaga SI, Kinashi Y, et al. Radiobiological evidence suggesting heterogeneous microdistribution of boron compounds in tumors: Its relation to quiescent cell population and tumor cure in neutron capture therapy. Int J Radiat Oncol Biol Phys 1996;34:1081-1086.

24. Ono K, Masunaga S, Suzuki M, et al. The combined effect of boronophenylalanine and borocaptate in boron neutron capture therapy for SCCVII tumors in mice. Int J Radiat Oncol Biol Phys 1999;43:431-436.

25. Fairchild RG, Slatkin DN, Coderre JA, et al. Optimization of boron and neutron delivery for neutron capture therapy. Pigment Cell Res 1989;2:309-318.

26. Setiawan Y, Halliday GM, Harding AJ, et al. Effect of L-10B-p boronophenylalanine-fructose and the boron neutron capture reaction on mouse brain dopaminergic neurons. Cancer Res 1995;55:874-877.

27. Capuani S, Gili T, Cametti C, et al. Radiowave dielectric investigation of boron compounds distribution in cultured tumour cells: Relevance to boron neutron capture therapy. Chem Phys Lett 2002;360:79-84.

28. Kageji T, Otersen B, Gabel D, et al. Interaction of mercaptoundecahydrododecaborate (BSH) with phosphatidylcholine: Relevance to boron neutron capture therapy. Biochim Biophys Acta 1998;1391:377-378.

29. Capala J, Makar MS, Coderre JA. Accumulation of boron in malignant and normal cells incubated in vitro with boronophenylalanine, mercaptoborane or boric acid. Radiat Res 1996;146: 554-560.

30. Papaspyrou M, Feinendegen EL, Müller-Gärtner HW. Preloading with L.Tyrosine Increases the uptake of boronophenylalanine in mouse Melanoma Cells. Cancer Res 1994;54: 6311-6314.

31. Wittig A, Sauerwein WA, Coderre JA. Mechanisms of transport of p-borono-phenylalanine through the cell membrane in vitro. Radiat Res 2000;153:173-180.

32. Cotzias G. L-Dopa for Parkinsonism. N Engl J Med 1968;278: 630.

33. Hornykiewicz O. L-DOPA: From a biologically inactive amino acid to a successful therapeutic agent. Amino Acids 2002;23: $65-70$.
34. Bordi F, Cametti C, Paradossi G. High-frequency dielectric study of side-chain dynamics in poly(lysine) aqueous solutions. Biopolymers 2000;53:129-134.

35. Di Pierro D, Lazzarino G, Pastore FS, et al. Determination of boronophenylalanine in biological samples using precolumn o-phthalaldehyde derivatization and reversed-phase highperformance liquid chromatography. Anal Biochem 2000;284: 301-306.

36. Looyenga H. Dielectric constants of heterogeneous mixtures. Physica 1965;31:401-406.

37. Bredberg E, Lennemas H, Paalzow L. Pharmacokinetics of levodopa and carbidopa in rats following different routes of administration. Pharma Res 1994;11:549-555.

38. Kent AP, Stern GM, Webster RA. The effect of benserazide on the peripheral and central distribution and metabolism of levodopa after acute and chronic administration in the rat. $\mathrm{Br}$ J Pharmacol 1990;100:743-748.

39. Hsieh $\mathrm{CH}$, Chen YF, Chen FD, et al. Evaluation of pharmacokinetics of 4-borono-2-(18)F-fluoro-L-phenylalanine for boron neutron capture therapy in a glioma-bearing rat model with hyperosmolar blood-brain barrier disruption. $\mathrm{J} \mathrm{Nucl} \mathrm{Med}$ 2005;46:1858-1865.

40. Barth RF, Yang W, Bartus RT, et al. Neutron capture therapy of intracerebral melanoma: Enhanced survival and cure after blood-brain barrier opening to improve delivery of boronophenylalanine. Int J Radiat Oncol Biol Phys 2002;52:858-868.

41. Yang W, Barth RF, Rotaru JH, et al. Boron neutron capture therapy of brain tumors: Enhanced survival following intracarotid injection of sodium borocaptate with or without blood-brain barrier disruption. Int J Radiat Oncol Biol Phys 1997;37:663-672.

42. Joel DD, Coderre JA, Micca PL, Nawrocky MM. Effect of dose and infusion time on the delivery of p-boronophenylalanine for neutron capture therapy. J Neurooncol 1999;41:213-221.

43. Morris GM, Micca PL, Nawrocky MM, Weissfloch LE, Coderre JA. Long-term infusions of p-boronophenylalanine for boron neutron capture therapy: Evaluation using rat brain tumor and spinal cord models. Radiat Res 2002;158:743-752. 